\title{
A Mathematical Evaluation of Mitral Regurgitation Severity with EROA
}

\author{
Kalpana Saini ${ }^{1, *}$, M. L. Dewal ${ }^{1,}$, Manojkumar Rohit ${ }^{2}$ \\ ${ }^{1}$ Department of Electrical Engineering, Indian Institute of Technology Roorkee, Uttarakhand, 247667, India \\ ${ }^{2}$ Advanced Cardiac Center, Post Graduate Institute of Medical Education and Research, Chandigarh, India \\ *Corresponding Author: kal_2312@rediffmail.com
}

Copyright (C) 2013 Horizon Research Publishing All rights reserved.

\begin{abstract}
It is to be investigated and verified that vena contracta width (VCW) obtained from color Doppler images and effective regurgitant orifice area (EROA) obtained from the $\mathrm{VCW}$ is capable of quantifying the severity of mitral regurgitation (MR). A mathematical expression has been developed between VCW and EROA. Vena contracta width is an efficient method for grading the severity of MR, but it is very difficult to locate this narrowest part exactly in transthoracic echocardiography as well as in transesophageal echocardiography. So, indeed, there is a need to develop a method which is capable of finding out the vena contracta width. A comparison has also been made between the results obtained using the proposed computing method and the results obtained by the clinicians, manually. For this analysis have been done from the results obtained with automatic VCW finding method and with clinical data for severity grading.
\end{abstract}

Keywords Vena Contracta Width, Effective Regurgitant Orifice Area, Mitral Regurgitation, Severity Grading, Transthoracic Echocardiography, Transesophageal Echocardiography

\section{Introduction}

At present interest in color Doppler imaging for vena contracta for the assessment of mitral re gurgitation is increasing progressively [1], [2] and [3]. The vena contracta is the narrowest part of the regurgitant jet, which can also be called as 'neck' of the jet [4] and having location just downstream from the orifice and before the jet area [5].

The cross sectional area is directly related to the regurgitant orifice area. Due to the complex and the unpredictable shape, it is very difficult to measure the regurgitant orifice area. For such a measurement jet is to be imaged in the short axis plane. In clinical settings, it is very difficult to localize the narrowest area from the jet. So a correlation is measured between the $\mathrm{VCW}$ which is measured from the zoomed view of the parasternal long axis and the apical views, and the EROA, by quantitative Doppler [3]. Relationship between the EROA and the VCW given at many places in the literatures [6], [7] and [8]. This relationship shows good results even in eccentric MR [9]. The VCW is the one dimensional measurement whereas EROA is the two dimensional so there are some limitations also. But in [10] it shown that VCW is the much better tool for measurement of severity of MR. Such as VCW less than $0.3 \mathrm{~cm}$ indicates the mild MR. If this value is in between 0.3 and 0.5 then it is the case of moderate MR. But if the VCW is more. than $0.5 \mathrm{~cm}$ than it indicates the severe MR.

In any view the localization of the VCW is very difficult. So an experienced clinician is required for the measurement of such a narrowest portion. This gets very time consuming also to locate the exact VC. Hence as discussion in our research paper [14] an automatic segmentation have been done of echocardiographic images to get VCW. In this paper, a mathematical solution is derived which gives a relation between VCW and EROA.

\section{Mathematical Treatment for EROA Calculation}

Mathematically, blood flow can be described by Darcy's law (which can be viewed as the fluid equivalent of Ohm's law) and approximately by Hagen-Poiseuille equation:

$$
\begin{gathered}
\text { Darcy'slaw } F=\Delta P / R \\
\text { Hagen-PoiseuilleEquation } R=\left(v L / r^{4}\right)(8 / \pi)
\end{gathered}
$$

Where $F$ is blood flow, $P$ is pressure, $R$ is resistance, $v$ is blood viscosity, $L$ is length of tube, and $r$ is radius of tube.

Here we are trying to calculate the vena contracta width according to the explanation given in [11]. However they used their formulation for the fluid flow. Here motive is to find out the vena contract width formulation for the mitral regurgitation jet. 
When blood passes through the mitral orifice then the momentum flux is given as:

$$
\frac{d p}{d t}=\rho\left(v_{\text {atr }}^{3} A_{\text {vena }}-v_{\text {vent }}^{2} A_{\text {vent }}\right) \approx \rho v_{\text {vena }}^{2} A_{\text {vena }} \approx 2 P_{\text {vent }} A_{\text {vena }}
$$

Where $A_{\text {vent }}, A_{\text {atr }}$ and $A_{\text {vena }}$ are the area of jet in ventricle, atrium and vena contracta, $v_{\text {vent }}, v_{\text {atr }}$ and $v_{\text {vena }}$ are the velocities of the blood in the ventricle, atrium and vena contracta, and $P_{\text {vent }}, P_{\text {atr }}$ and $P_{\text {vena }}$ are the pressures in the respective regions.

According to Bernoulli's equation in the limit that $P_{\text {vena }} \ll<P_{\text {vent }}$. The force that causes this momentum change is:

$$
\begin{aligned}
& F \approx P_{\text {vent }} A_{\text {vent }}-\left[P_{\text {vent }}\left(A_{\text {vent }}-A_{\text {atr }}\right)+P_{\text {vena }} A_{\text {vena }}\right] \\
& =P_{\text {vent }} A_{\text {atr }} \approx P_{\text {vent }} A_{\text {atr }}
\end{aligned}
$$

Vena contracta can be estimated as:

$$
A_{\text {vena }}=\frac{A_{\text {atr }}}{2}
$$

K.T McDonald [11] refer the comment given by Maxwell [12] on the vena contracta, he suggest that if vena contracta of the jet is to be measured in two dimensional potential flow through the orifice (in present case mitral orifice) then a more accurate analysis could be done based on the velocity potential $\phi$ that exists when the flow is irrotational.

Any analytic function $w(z)=\phi+i \psi$ of the complex varable $z=x+i y$, obeys the Cauchy-Riemann equations,

$$
\frac{\partial \phi}{\partial x}=\frac{\partial \psi}{\partial y}
$$

and

$$
\frac{\partial \phi}{\partial y}=-\frac{\partial \psi}{\partial x}
$$

Which imply that $\nabla^{2} \phi=0=\nabla^{2} \psi$. That is, any analytic function of a complex variable can, in principle, be related to the velocity potential of the blood flow. Furthermore, the curves $\psi(x, y)=$ constant follow streamlines of the Potential $\phi(x, y)$ (and vice versa).

According to Kirchhoff [13] if the blood flow includes a free surface, on which the pressure is constant, then Bernoulli's equation implies that the velocity of the blood flow is constant on this surface.

This velocity can always be scaled to unity. Then, since, $V=-\nabla \phi=-(\partial \phi / \partial x, \partial \phi / \partial y)$ the scaled flow on a free surface obeys

$$
\left(\frac{\partial \phi}{\partial x}\right)^{2}+\left(\frac{\partial \phi}{\partial y}\right)^{2}=1
$$

Furthermore, according to the Jacobian transformation of area elements

$$
d x d y=J d \phi d \psi=J J^{\prime} d x d y,
$$

So, $J J^{\prime}=1$. The Cauchy-Riemann equations for $\phi+i \psi=w(x+i y)$ and $x+i y=w^{-1}(\phi+i \psi)$ allow us to write the Jacobian determinants as

$$
J=\frac{\partial \phi}{\partial x} \frac{\partial \psi}{\partial y}-\frac{\partial \phi}{\partial y} \frac{\partial \psi}{\partial x}=\left(\frac{\partial \phi}{\partial x}\right)^{2}+\left(\frac{\partial \phi}{\partial y}\right)^{2}
$$

and

$$
J^{\prime}=\left(\frac{\partial x}{\partial \phi}\right)^{2}+\left(\frac{\partial y}{\partial \phi}\right)
$$

Then, the scaled flow on a free surface also obeys

$$
\left(\frac{\partial x}{\partial \phi}\right)^{2}+\left(\frac{\partial y}{\partial \phi}\right)^{2}=1
$$

Kirchhoff [3], extended Helmholtz' technique of functions $w(z)$, that are defined implicitly via knowledge of $z(w)$ by consideration of the derivative, $d z / d w$ defined as

$$
\frac{d z}{d w}=-f(w)-\sqrt{f^{2}(w)-1},
$$

arguing that if along some portion of a streamline $\psi$ $=$ constant the function $f$ is real and $|f|$ less than 1 , then $d x / d \phi=\operatorname{Re}(d z / d w)=-f \quad$ and $\quad d y / d \phi=\operatorname{Im}(d z / d w)=-\sqrt{1-f^{2}}$, such that eq. (10) is satisfied, and this portion of the streamline corresponds to a free surface of the blood flow. In particular, the function

$$
f=e^{-w}=e^{-\phi} \cos \psi-i e^{-\phi} \sin \psi
$$

obeys eq. (10) for $\psi=0$ and $\phi>0$. The free surface is defined by the equations

$$
\frac{d x}{d \phi}=-f=e^{-\phi}
$$

and

$$
\frac{d y}{d \phi}=-\sqrt{1-f^{2}}=-\sqrt{1-e^{-2 \phi}}(\phi>0, \psi=0)
$$

which integrate to

$$
x=e^{-\phi}-1
$$

and

$$
y=\sqrt{1-e^{-2 \phi}}-\ln \left(e^{\phi}+\sqrt{e^{2 \phi}-1}\right)(\phi>0, \psi=0)
$$

Similarly, the streamline $\psi=\pi$ also obeys eq. (10) for 
$\phi>0$, such that

$$
\frac{d x}{d \phi}=e^{-\phi}
$$

and

$$
\frac{d y}{d \phi}=-\sqrt{1-e^{-2 \phi}}(\phi>0, \psi=\pi)
$$

which integrate to

$$
x=-x_{0}+1-e^{-\phi}
$$

and

$$
y=\sqrt{1-e^{-2 \phi}}-\ln \left(e^{\phi}+\sqrt{e^{2 \phi}-1}\right)(\phi>0, \psi=\pi)
$$

On this streamline $d z / d \phi$ is real and negative for $\phi<0$, so $y$ is again constant at 0 while $x$ decreases from $-x_{0}$ to $-\infty$.

Furthermore, on the streamline $\psi=\pi / 2, d z / \phi$, is purely imaginary so that $x$ is constant (at $-x_{0} / 2$ ), and this streamline is the symmetry axis of the flow.

The constant $x_{0}$ can be determined by noting that for large negative $y$ the streamlines $0 \leq \psi \leq \pi$ are all in the $y$ direction, so the corresponding equipotentials of $\phi$ are at constant $y$. Then, the velocity $V=-\nabla \phi$ is constant across the jet, with value unity, such that integrating across the jet at large negative $y$ find, using the Cauchy-Riemann eq.(6)

$$
\Delta \psi=\pi=\int_{-x_{0}+1}^{-1} \frac{\partial \psi}{\partial x} d x=-\int_{-x_{0}+1}^{-1} \frac{\partial \phi}{\partial y} d x=v \int_{-x_{0}+1}^{-1} d x=x_{0}-2
$$

and hence the width of the mitral orifice is $x_{0}=\pi+2$. Thus, we determine the vena contract a to be

Table 1. MR Severity Checking On the Basis of VCW

\begin{tabular}{|c|c|c|c|c|}
\hline Patient & $\begin{array}{c}\text { Vena Contracta } \\
\text { width in (pixels) } \\
\text { by Automatic VC } \\
\text { width detection } \\
\text { method }\end{array}$ & $\begin{array}{c}\text { Vena Contracta } \\
\text { width (cm) by } \\
\text { Automatic VC } \\
\text { width detection } \\
\text { method }\end{array}$ & $\begin{array}{c}\text { MR Severity } \\
\text { (Automatic VC } \\
\text { width method) }\end{array}$ & $\begin{array}{c}\text { MR Severity } \\
\text { (by } \\
\text { Clinicians) }\end{array}$ \\
\hline 1 & 13 & $\mathbf{0 . 5 8}$ & severe & severe \\
\hline 2 & 14 & $\mathbf{0 . 6 1}$ & severe & severe \\
\hline 3 & 8 & $\mathbf{0 . 4 6}$ & moderate & moderate \\
\hline 4 & 12 & $\mathbf{0 . 5 6}$ & severe & severe \\
\hline 5 & $\mathbf{8}$ & $\mathbf{0 . 4 6}$ & moderate & moderate \\
\hline 6 & 12 & $\mathbf{0 . 5 6}$ & severe & severe \\
\hline 7 & 11 & $\mathbf{0 . 5 4}$ & severe & severe \\
\hline 8 & 11 & $\mathbf{0 . 5 4}$ & severe & severe \\
\hline 9 & 9 & 0.39 & moderate & moderatc \\
\hline
\end{tabular}

$$
A_{\text {vena }}=\frac{\pi}{\pi+2} A_{\text {mitral orifice }}=0.61 A_{\text {mitral orifice }}
$$

$A_{\text {mitral orifice }}$, is effective regurgitant orifice area (EROA), because this is the case when the orifice is considered as a circular aperture but actually mitral orifice is not circular.
If $\mathrm{VCW}$ is represented as $W_{\text {vena }}$ and mitral orifice diameter is $d_{\text {mitral orifice }}$ and we know that

Table 2. MR Severity Cheking On the Basis of EROA

\begin{tabular}{|c|c|c|c|}
\hline Patient & $\begin{array}{c}\text { Effective Regurgitant Orifice } \\
\text { Area (EROA) in } \mathbf{c m}^{2} \text { (by } \\
\text { Proposed method ) }\end{array}$ & $\begin{array}{c}\text { MR Severity (by } \\
\text { Proposed EROA } \\
\text { method) }\end{array}$ & $\begin{array}{c}\text { MR Severity } \\
\text { (by Clinicians) }\end{array}$ \\
\hline 1 & 0.43 & severe & severe \\
\hline 2 & 0.47 & severe & severe \\
\hline 3 & 0.26 & moderate & moderate \\
\hline 4 & 0.39 & moderate-severe & severe \\
\hline 5 & 0.26 & moderate & moderate \\
\hline 6 & 0.39 & moderate-severe & severe \\
\hline 7 & 0.37 & moderate-severe & severe \\
\hline 8 & 0.37 & moderate & moderate \\
\hline 9 & 0.20 &
\end{tabular}

$$
\begin{gathered}
\pi W_{\text {vena }}^{2}=0.61 \pi d_{\text {mitral orifice }}^{2} \\
W_{\text {vena }}^{2}=\left(0.78 d_{\text {mitralorifice }}\right)^{2} \\
d_{\text {mitralorifice }}=\frac{W_{\text {vena }}}{0.78}
\end{gathered}
$$

\section{Results}

Table 1 shows the values of vena contracta width of different echocardiographic images which are taken from the patients suffering from mitral regurgitation. These results are obtained from automatic vena contracta width detection method. The results corroborate quite closely with the severity grades demined by the clinicians. If the cases 1, 2, 4, 6,7 and 8 are viewed, the values of vena contracta width are greater than $0.5 \mathrm{~cm}$ suggesting that the MR is of the severe grade. Whereas, in cases 3, 5, and 9, the VCWs observed are within 0.4 to $0.5 \mathrm{~cm}$ wide. This range of VCW suggests that the MR is of the moderate severity grade. The MR severity evaluation using proposed method corroborates $100 \%$ with MR severity gradation carried out by the clinicians.

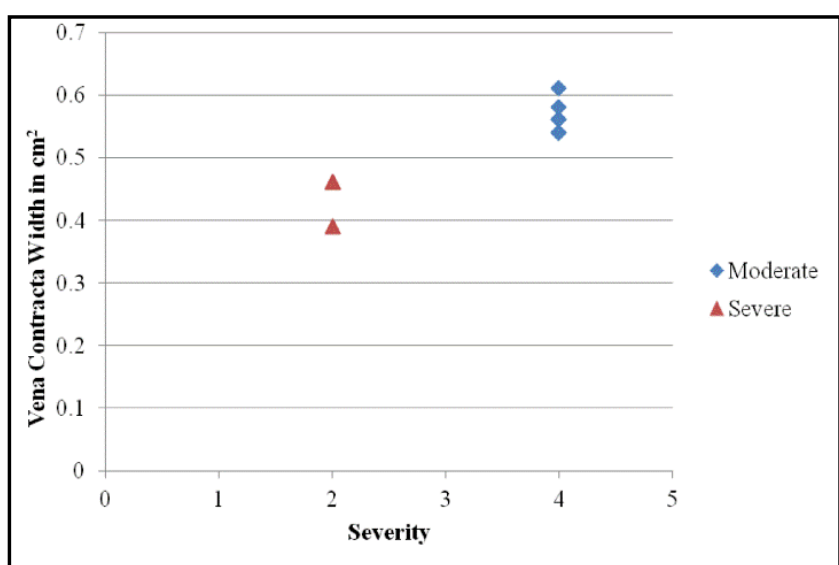

Figure 1. Grading result by VCW. The criteria for grading severity are: Mild VCW $<0.3 \mathrm{~cm}$, Moderate $0.3 \mathrm{~cm}$ to $0.49 \mathrm{~cm}$, Severe VCW $\geq 0.5 \mathrm{~cm}$ 


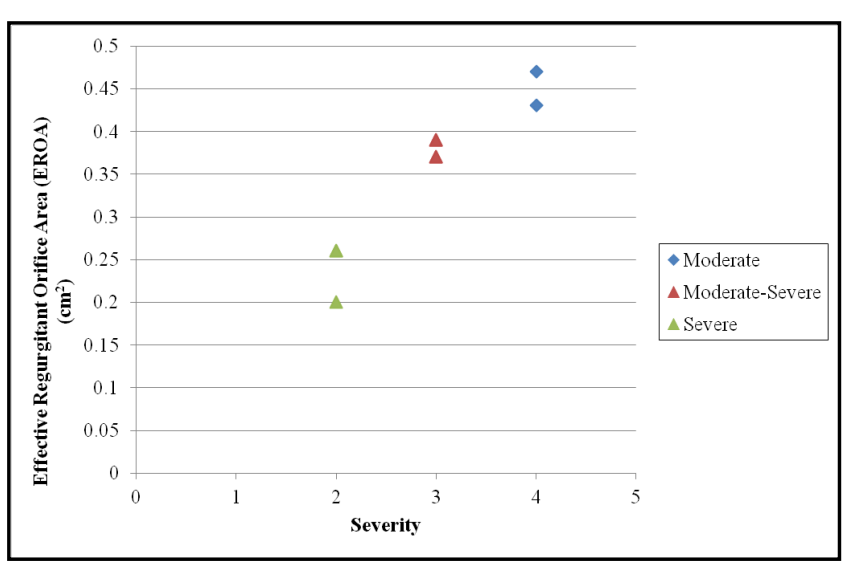

Fig.ure 2. Grading of results by EROA. The criterion for severity grading are, mild EROA $<0.2 \mathrm{~cm} 2$, moderate EROA $0.2-0.29,0.3-0.39 \mathrm{~cm} 2$, Severe EROA $\geq_{0.4 \mathrm{~cm} 2}$

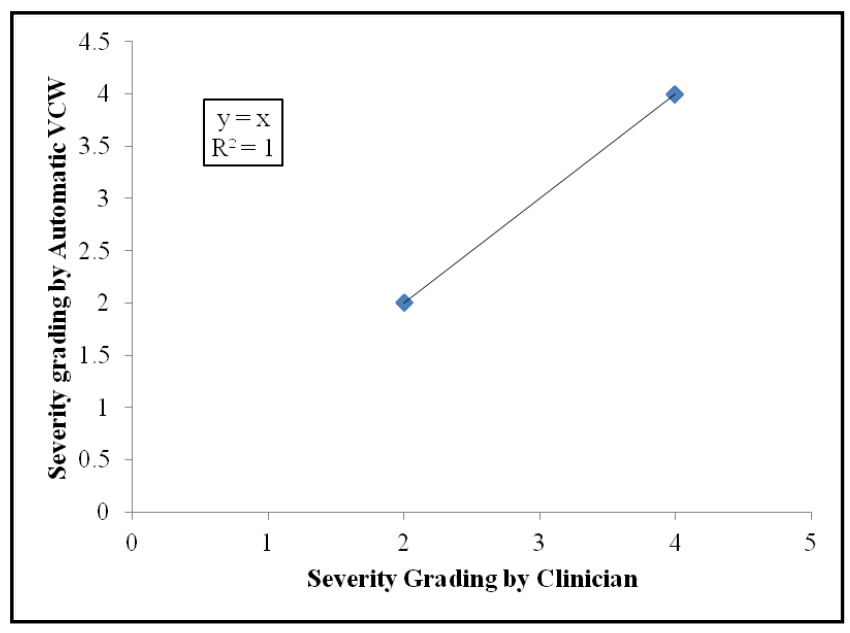

Figure 3. Linear regression plots showing exact correlation between severity by automatic VCW and by clinicians

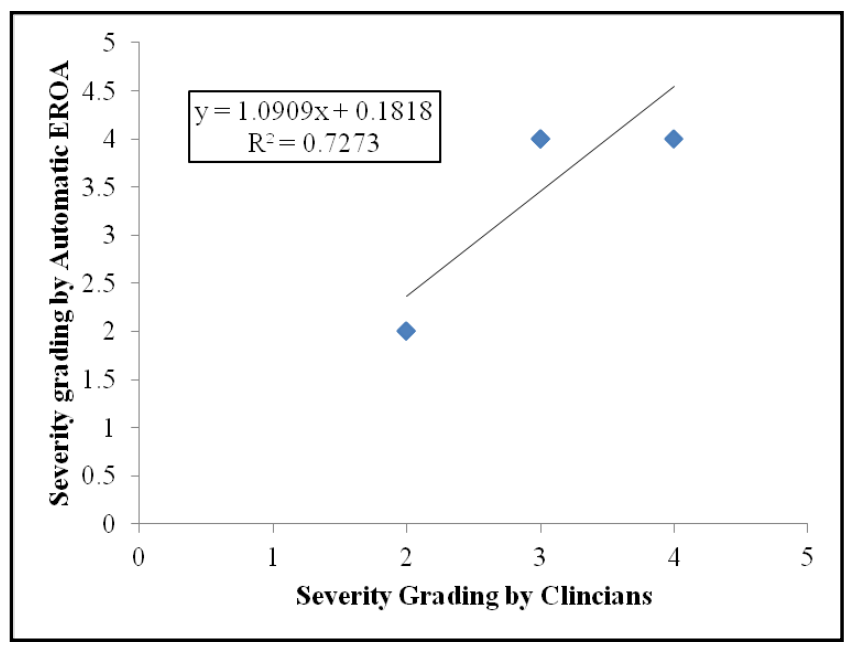

Figure 4. Linear regression plots showing good correlation between severity by automatic EROA and by clinicians

From the values of vena contract a width obtained from the table 1, EROA can be calculated using our proposed method. Results are shown in table 2. We know that EROA below $0.2 \mathrm{~cm}^{2}$ shows the mid MR, however between $0.2 \mathrm{~cm}^{2}$ to $0.4 \mathrm{~cm}^{2} \mathrm{MR}$ is categorized as moderate MR. If this value goes beyond $0.4 \mathrm{~cm}^{2}$, than MR will be sever MR. here we create a more section for severity grading is moderate-severe.

\section{Statistical Analysis of Results}

The grading result of MR, by VCW is shown by Fig.1. The grading result is that out of nine patients 3 patients are graded as moderate $(0.437 \pm 0.023 \mathrm{~cm})$ and 6 patients as severe $(0.565 \pm 0.045 \mathrm{~cm})$.

The grading result of MR, by EROA is shown by Fig.2. The grading result is that out of nine patients 3 patients are graded as moderate $(0.24 \pm 0.02 \mathrm{~cm})$ and 4 patients as moderate-severe $(0.38 \pm 0.01 \mathrm{~cm})$, and 2 patients as severe $(0.45 \pm 0.02 \mathrm{~cm})$.grading by clinician and severity grading by automatic effective regurgitant orifice area. The result reveals that severity grading by EROA correlates significantly to severity grading by clinician $\left(\mathrm{r}^{2}=0.7273\right)$.

Fig.5 shows the correlation between vena contract a width and effective regurgitant orifice area. There is a good correlation between EROA and vena contract width ( $\mathrm{r} 2$ $=0.9874$ ).

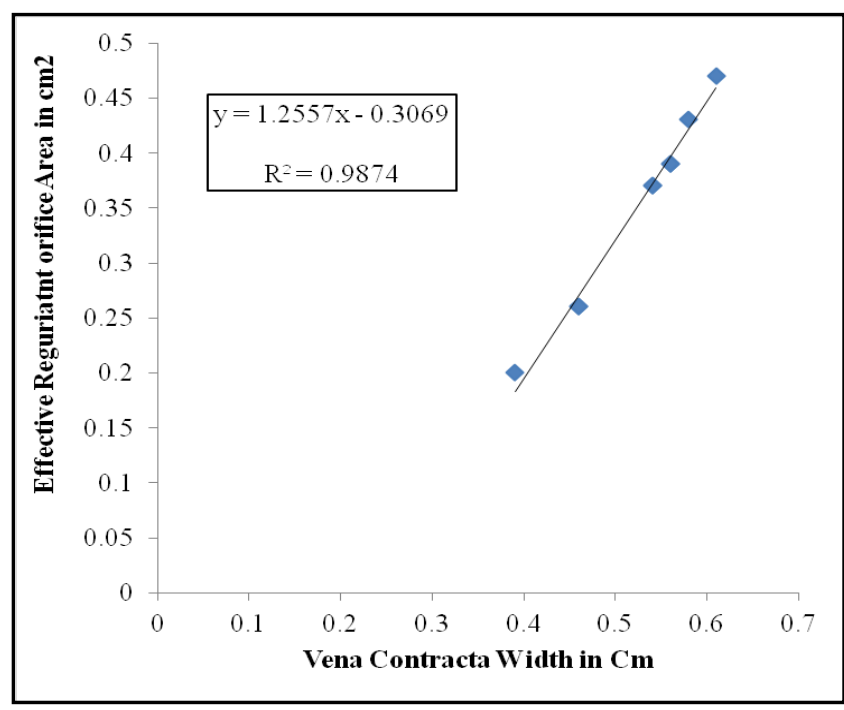

Figure 5. Linear regression plots showing exact correlation between severity by automatic VCW and EROA

\section{Conclusion}

Vena contract a diameter is directly related to the effective regurgitant orifice area (EROA). Since, the algorithm of the proposed method is quit fast, the multiple images during the systole can be images and dynamicity of EROA can be examined. Statistical results shows good correlation between severity grading proposed by clinicians and by the automatic VCW method and EROA method.

\section{REFERENCES}


[1] C. Tribouilloy, W.F. Shen, J. P. Quere, J. L. Rey, D. Choquet, H. Dufosse, J.P Lesbre, "Assessment of Severity of Mitral Regurgitation by Measuring Regurgitant Jet Width at its Origin with Transesophageal Doppler Color Flow Imaging", Circulation, vol. 85, pp.1248 -1253, 1992.

[2] S. A. Hall, M. E. Brickner, D. L. Willett, W. N. Irani, I. Afridi, P. A. Grayburn, "Assessment of Mitral Regurgitation Severity by Doppler Color Flow Mapping of the Vena Contracta", Circulation, vol 9, pp. 636-642, 1997.

[3] M. Ishii, M. Jones, T. Shiota, I. Yamada, R. S. Heinrich, S. R. Holcomb, A. P. Yoganathan, D. J. Sahn, "Quantifying Aortic Regurgitation by Using the Color Doppler-Imaged Vena Contracta: A Chronic Animal Model Study", Circulation, vol. 96, pp. $2009-2015,1997$.

[4] T. Irvine, X. K. Li, D. J. Sahn, A. Kenny, "Assessment of Mitral Regurgitation", Heart, vol. 88 (Suppl IV), pp. iv11-iv19, 2002.

[5] M. Ishii, M. Jones, T. Shiota, I. Yamada, R. S. Heinrich, S. R. Holcomb, A. P. Yoganathan, D. J. Sahn, "Evaluation of Eccentric Aortic Regurgitation by Color Doppler Jet and Color Doppler-Imaged Vena Contracta Measurements: An Animal Study of Quantified Aortic Regurgitation", Am Heart $J$, vol .132, pp. 796-804, 1996.

[6] P. Grayburn, W. Fehske, H. Omran, M. E.Brickner, B. Lüderitz, "Multiplane Transesophageal Echocardiographic Assessment of Mitral Regurgitation by Doppler Color Flow Mapping of the Vena Contracta" , Am J Cardiol, vol. 74, pp.912-917, 1994.

[7] W. Fehske , H. Omran , M. Manz , J. Köhler, A. Hagendorff, B. Lüderitz, "Color-Coded Dopplerimaging of the Vena
Contracta as a Basis for Quantification of Pure Mitral Regurgitation", Am J Cardiol, vol. 73 pp. 268-274, 1994.

[8] S. K. Heinle, S. A. Hall, M. E. Brickner, D. L.Willett, P. A. Grayburn, "Comparison of Vena Contracta Width by Multiplane Transesophageal Echocardiography with Quantitative Doppler Assessment of Mitral Regurgitation", Am J Cardiol, vol. 81, pp.175-179, 1998.

[9] X. Zhou, M. Jones, T. Shiota, I. Yamada, D. Teien, D. J. Sahn, "Vena Contracta Imaged by Doppler Color Flow Mapping Predicts the Severity of Eccentric Mitral Regurgitation Better than Color Jet Area: A Chronic Animal Study", J Am Coll Cardiol, vol. 30, pp. 1393-1398, 1997.

[10] S. Hall, M. Brickner, D. Willett, et al., "Assessment of Mitral Regurgitation by Doppler Color Flow Mapping of the Vena Contracta", Circulation, vol. 95, pp.636-642, 1997.

[11] K. T. McDonald, Vena Contracta (Feb. 16, 2005) $\mathrm{http}: / /$ www.hep.princeton.edu/ mcdonald/mumu/target/pump/vena contracta.pdf.

[12] J.C. Maxwell, "Remarks on the preceding Paper by G.O. Hanlon on the Vena Contracta" ,Proc. London Math. Soc., vol. 3 , no. 6,1869 .

[13] G. Kirchhoff, "Zur Theorie frier Fl"ussigkeitsstrahlen", $J$. Math. vol. 70, no. 37, 1869.

[14] K. Saini, M. L. Dewal, M. Rohit, "Segmentation of Mitral Regurgitant Jet Using the Combination of Wavelet and Watershed Transformation", 8th IEEE Colloquium on Signal Processing \& Its Applications, Melaka, Malaysia, pp. 74- 79, 23-25th March 2012. 